\title{
PERFIL CLÍNICO E SOCIODEMOGRÁFICO DE PACIENTES COM DEFICIÊNCIA VISUAL E DIABETES MELLITUS
}

\section{CLINICAL AND SOCIODEMOGRAPHIC PROFILE OF PATIENTS WITH VISION DISORDERS AND DIABETES MELLITUS}

\section{PERFIL CLÍNICO Y SOCIODEMOGRÁFICO DE PACIENTES CON TRASTORNOS VISUALES Y DIABETES MELLITUS}

Evandro Scarso de Brito ${ }^{1}$, Maria Helena Pinto ${ }^{2}$, Denise Beretta ${ }^{2}$, Daniele Alcalá Pompeo ${ }^{2}$, Thiare Júnia de Francisco Brito ${ }^{5}$, Rildo César Nunes Czorny 6

\section{RESUMO}

Objetivo: Descrever e analisar as características clínicas e sociodemográficas de pacientes deficientes visuais diabéticos. Método: Estudo transversal, descritivo, desenvolvido em um Centro de Reabilitação Visual com 51 pacientes deficientes visuais diabéticos que participaram de uma entrevista estruturada. Foi realizada análise descritiva das variáveis, testou-se a normalidade pelo teste de Kolmogorov Smirnov, e a análise bivariada entre a variável dependente e as variáveis independentes utilizando o teste estatístico Regressão de Poisson. As associações foram consideradas estatisticamente significativas quando $p<0,05$. Resultados: A maioria dos participantes era do sexo masculino (58,8\%), com 60 anos ou mais $(43,1 \%)$, branco $(49,0 \%)$ e sem companheiro $(56,8 \%)$. Os portadores de diabetes tipo 2 (54,9\%) foram diagnosticados, há mais de dez anos (56,9\%), apresentavam sobrepeso (43,15\%) e eram hipertensos (74,5\%). A maior parte da população entrevistada era cega (72,5\%) e relatou que as doenças oculares foram a causa da deficiência visual. Entre os diabéticos, $47 \%$ dos pacientes tipo 1 apresentavam retinopatia diabética. Conclusão: A caracterização clínica e sociodemográfica dos pacientes deficientes visuais norteiam o gerenciamento do cuidado e os programas de promoção e prevenção a serem desenvolvidos, uma vez que as estratégias de controle das doenças sofrem diferentes influências, dependendo das características da população acometida.

DESCRITORES: Características da população; Diabetes Mellitus; Adulto; Idoso; Transtornos da visão.

\section{ABSTRACT}

Objective: To describe and analyze the clinical and sociodemographic characteristics of diabetic and visually impaired patients. Method: A cross-sectional and descriptive study was developed in a visual rehabilitation center with 51 diabetic and visually impaired patients who participated in a structured interview. The descriptive analysis of the variables was performed and normality was tested by the Kolmogorov Smirnov test. Bivariate analysis between the dependent variable and the independent variables was performed using Poisson regression. The associations were considered statistically significant when $p<0.05$. Results: Most participants were men (58.8\%), aged 60 years old or older (43.1\%), white (49.0\%) and had no partner (56.8\%). Patients with type-2 diabetes (54.9\%) were diagnosed over 10 years ago (56.9\%) and were overweight (43.15\%) and hypertensive (74.5\%). Most of the interviewed population was blind (72.5\%) and reported that ocular diseases were the cause of visual impairment. Among the diabetic patients, $47 \%$ of type- 1 patients had diabetic retinopathy. Conclusion: The clinical and sociodemographic characterization of visually impaired patients guide the management of care and the promotion and prevention programs to be developed, since the disease control strategies suffer different types of influence, depending on the characteristics of the affected population.

DESCRIPTORS: Population Characteristics; Diabetes Mellitus; Adult; Aged; Vision Disorders.

\section{RESUMEN}

Objetivos: Describir y analizar las características clínicas y sociodemográficas de pacientes con deficiencia visual y diabetes. Método: Estudio transversal, descriptivo, desarrollado en un centro de rehabilitación visual en 51 pacientes con trastornos visuales y diabetes que participaron en una entrevista padronizada. Se realizó un análisis descriptivo de las variables, se comprobó la normalidad aplicando test de Kolmogorov-Smirnov, y un análisis bivariado entre variables dependientes e independientes, utilizando la Regresión de Poisson. Las asociaciones fueron consideradas como estadísticamente significantes con $\mathrm{p}<0,05$. Resultados: La mayoría de los participantes eran de sexo masculino $(58,8 \%)$, de 60 años o mayores $(43,1 \%)$, blancos $(49,0 \%)$ y sin pareja $(56,8 \%)$. Los afectados por diabetes tipo $2(54,9 \%)$ tenían un diagnóstico mayor a 10 años (56,9\%), mostraban sobrepeso (43,15\%) y eran hipertensos (74,5\%). La mayoría de los entrevistados eran ciegos (72,5\%) e informaron que las enfermedades oculares provocaron su trastorno visual. Entre los diabéticos, el $47 \%$ de los pacientes tipo 1 presentó retinopatía diabética. Conclusión: La caracterización clínica y sociodemográfica de los pacientes con trastornos visuales sugiere el gerenciamiento del cuidado y de los programas de promoción y prevención a desarrollarse, considerando que las estrategias de control de las enfermedades reciben diferentes influencias, dependiendo de las características de la población que las padece.

Descriptores: Características de la Población; Diabetes Mellitus; Adulto; Anciano; Trastornos de la Visión.

${ }^{1}$ Enfermeiro. Especialista. Mestrando do Programa de Pós Graduação em Enfermagem da Faculdade de Medicina de São José do Rio Preto. ${ }^{2}$ Enfermeira. Doutora. Docente do curso de Graduação em enfermagem da Faculdade de Medicina de São José do Rio Preto. ${ }^{3}$ Terapeuta Ocupacional. Especialista. Profissional do Instituto Riopretense de Cegos Trabalhadores. ${ }^{4}$ Enfermeiro. Mestre. Docente do curso de Graduação em enfermagem da União das Faculdades dos Grandes Lagos.

Como citar este artigo:

Brito ES, Pinto MH, Beretta D, et al. Perfil clínico e sociodemográfico de pacientes com deficiência visual e diabetes mellitus. Revista de Enfermagem do Centro-Oeste Mineiro. 2019;9:e3475. [Acess___ Disponível em: ___ DOI: HTTP://dx.doi.org/10.19175/recom.v9i0.3475 


\section{INTRODUÇÃO}

O Diabetes Mellitus (DM) é um distúrbio metabólico complexo, heterogêneo, caracterizado por aumento da concentração de glicose no sangue decorrente da resistência à ação da insulina, secreção insuficiente de insulina ou a ambos. A classificação e diagnóstico do DM são complexos e têm sido objeto de muito estudo, debate e revisão, que se estendem sobre muitas décadas. O DM pode ser classificado em três tipos principais: tipo 1, tipo 2 e gestacional ${ }^{(1)}$.

O DM está associado a fatores socioeconômicos, demográficos e a outras condições de saúde passíveis de intervenção, salientando a necessidade de políticas públicas mais efetivas voltadas, principalmente, para a modificação de hábitos de vida ${ }^{(2)}$. A rápida urbanização, transição epidemiológica, transição nutricional, estilo de vida sedentário, excesso de peso, crescimento e envelhecimento populacional são fatores que contribuem para 0 aumento da prevalência do $\mathrm{DM}^{(3)}$.

De acordo com estimativas da International Diabetes Federation (IDF), no mundo, em 2017, o DM acometeu 326,5 milhões de pessoas, em idade ativa (20-64 anos) e 122,8 milhões de pessoas entre 65 a 99 anos. Em 2045, estima-se que o número de pessoas, em idade ativa com DM, aumentará para 438,2 milhões, e as pessoas entre 65 e 99 anos aumentarão para 253,4 milhões. No Brasil, em 2017, o DM acometeu 12,5 milhões de pessoas, em idade ativa, com projeção de 21,8 milhões em $2045^{(1)}$.

Com o atual envelhecimento populacional do Brasil, o DM certamente passará a ter maior contribuição, para a mortalidade no país, aumentando mais de 400 vezes a faixa etária de 0 a 29 anos para 60 anos ou mais ${ }^{(3)}$.

Aproximadamente, quatro milhões de pessoas, no mundo, com idade entre os 20 e 79 anos, morreram em razão do DM, em 2017, o que equivale a uma morte a cada oito segundos. Considerando essa mesma faixa etária, o impacto econômico, para os países, sistemas de saúde e, acima de tudo, para os indivíduos com DM e seus familiares, aumentou de US \$ 232 bilhões, em 2007, para US $\$ 727$ bilhões, em 2017, em todo o mundo ${ }^{(1)}$.

Considerando o rápido envelhecimento da população brasileira, é importante o investimento em programas de promoção, visando a intervenções precoces, para prevenir e controlar o DM, tais como: alimentação saudável, restrição do tabaco e consumo de álcool e programas para a prática de atividade física. Assim contribuirá na redução do sobrepeso e obesidade e outros fatores de risco comuns às Doenças Crônicas Não Transmissíveis (DCNT), como o $\mathrm{DM}^{(4)}$.

Os pacientes portadores de DM, caso não tratem ou controlem a doença, podem desenvolver mais complicações ${ }^{(3)}$ e possuem 30 vezes mais chances de se tornarem cegos que pacientes sem a doença ${ }^{(5)}$.

A deficiência visual (DV) é caracterizada pela perda total (cegueira) ou parcial (baixa visão ou visão subnormal) da visão, podendo ser congênita ou adquirida ${ }^{(6)}$. Estima-se que, no mundo, mais de 407 milhões de pessoas possuem algum tipo de DV, sendo que, dentre elas, 36 milhões de pessoas são cegas, mais de 216 milhões possuem DV moderada a grave e 188 milhões possuem comprometimento visual leve ${ }^{(7)}$.

No Brasil, o último Censo Demográfico, realizado em 2010, apontou que mais de 45 milhões de brasileiros apresentam, pelo menos, uma deficiência $(23,9 \%$ da população total), sendo que a DV foi a deficiência com maior ocorrência ( $18,6 \%$ da população brasileira) entre os quais $3,46 \%$ com DV grave ${ }^{(8)}$.

Existem evidências de intervenções eficazes visando melhorar o controle do DM e reduzir seus fatores de risco modificáveis, entretanto existem lacunas significativas na base de conhecimento sobre a temática. Em virtude do exposto e da escassez de artigos que descrevem e analisam o perfil clínico e sociodemográfico de pacientes com DV e DM, o objetivo deste estudo foi descrever e analisar as características clínicas e sociodemográficas dos pacientes deficientes visuais diabéticos.

\section{MÉTODO}

Trata-se de um estudo transversal, descritivo, quantitativo, desenvolvido no Centro de Reabilitação Visual, localizado no interior do Estado de São Paulo. A instituição atende pessoas com DV, referenciadas pelos estabelecimentos vinculados ao Sistema Único de Saúde (SUS); atende pacientes de todas as fases do ciclo de vida e possui, atualmente, 241 matriculados, 113 são adultos e idosos, dentre os quais 62 portadores de DM.

A população do estudo foi constituída de 62 pacientes adultos e idosos, com DV, matriculada na instituição, com diagnóstico de DM. Foram considerados elegíveis os pacientes 
que atenderam os seguintes critérios: ter idade igual ou superior a 18 anos, aceitar participar da pesquisa, assinar o Termo de Consentimento Livre e Esclarecido (TCLE) e estar frequentando regularmente a instituição. Foram excluídos onze pacientes, sendo cinco por não estarem com frequência regular na instituição, cinco por terem recusado participar da pesquisa e um paciente por ter falecido, ficando a amostra constituída de 51 pacientes.

A coleta de dados foi realizada de junho a agosto de 2018. Inicialmente, foi agendado um encontro com os pacientes selecionados para a apresentação do objetivo do estudo e das suas etapas. Neste encontro, foram entregues a cada um dos participantes duas vias do TCLE, previsto na Resolução no466/12 do Conselho Nacional de Saúde (CNS) e realizada a leitura por meio de um dispositivo portátil e independente que amplia e lê em alto som materiais impressos para indivíduos com DV; após o aceite, foi devolvida uma via do termo assinado, de forma imediata. Em seguida, realizou-se uma entrevista estruturada de forma individualizada e privativa, com duração média de 40 minutos, seguindo um instrumento de coleta de dados, elaborado pelo pesquisador, que contemplava as variáveis: perfil sociodemográfico (sexo, idade, estado civil, cor autodeclarada, escolaridade, atividade remunerada e renda mensal), perfil clínico e estilo de vida (tipo de DM, tempo de descoberta da doença, antecedentes familiares, tipos e adesão ao tratamento, dislipidemias, prática de exercícios físicos, tabagismo, uso de bebidas alcoólicas, hipertensão arterial sistêmica (HAS), acompanhamento na Atenção Primária à Saúde (APS), acesso a atividades educativas de prevenção ao DM, circunferência abdominal, Índice de Massa Corporal (IMC)) e aspectos relacionadas à DV.

Para a aferição do peso, foi utilizada uma balança portátil eletrônica, com capacidade de $200 \mathrm{~kg}$ e divisão de $50 \mathrm{~g}$. Os pacientes foram pesados com o mínimo de roupa possível e viável. Para a obtenção da estatura $(\mathrm{cm})$, utilizouse um estadiômetro portátil com extensão de dois metros e divisão de $0,1 \mathrm{~cm}$. Os pacientes ficaram em posição ereta, descalços e os tornozelos encostados no estadiômetro ${ }^{(9)}$.

Para medir a circunferência abdominal, utilizou-se uma fita métrica de $150 \mathrm{~cm}$, em que o paciente, em posição supina, inspirou profundamente e, ao final da expiração, realizouse a medida no maior perímetro abdominal entre a última costela e a crista ilíaca, segundo recomendações da $\mathrm{OMS}^{(9)}$.

O IMC foi usado, para caracterizar os pacientes sobre o estado nutricional e classificado, de acordo com as Diretrizes Brasileiras de Obesidade $(2016)^{(9)}$ : baixo peso < $18,5 \mathrm{~kg} / \mathrm{m}^{2}$; eutrófico entre 18,5 e $24,9 \mathrm{~kg} / \mathrm{m}^{2}$; sobrepeso entre 25 e $29,9 \mathrm{~kg} / \mathrm{m}^{2}$; obesidade I entre 30 e $34,9 \mathrm{~kg} / \mathrm{m}^{2}$; obesidade II entre 35 e $39,9 \mathrm{~kg} / \mathrm{m}^{2}$; obesidade III $>40 \mathrm{~kg} / \mathrm{m}^{2}$. Os pontos de corte da medida de circunferência abdominal adotados, para risco cardiovascular aumentado, foram: igual ou superior a $90 \mathrm{~cm}$, em homens e $80 \mathrm{~cm}$, em mulheres ${ }^{(9)}$.

Para a análise dos dados, utilizou-se o Software SPSS Statistics, versão 23, atrelado às funcionalidades da ferramenta Excel ${ }^{\circledR}$, versão 2016. Inicialmente, foi realizada uma análise descritiva das variáveis, sendo as quantitativas descritas em médias e desvios-padrão; e as qualitativas, em frequências simples $e$ percentuais. Testou-se a normalidade pelo teste de Kolmogorov Smirnov. Realizou-se a análise bivariada entre a variável dependente: "tipo de DM" e as variáveis independentes: "IMC", "circunferência abdominal", "tipo da DV", "tempo da DV", "causa/doença que levou à DV", "glaucoma", "catarata" e "Retinopatia Diabética $\mathrm{RD}^{\prime}$, empregando o teste estatístico Regressão de Poisson, que foi aplicado, para analisar a dependência de variáveis dicotômicas, quando cruzadas com variáveis dicotômicas $(2 \times 2)$ ou contínuas. Os testes contemplaram erro alfa de $5 \%$ e confiabilidade de $95 \%$, assim, as associações foram consideradas estatisticamente significativas quando $p<0,05$.

Este estudo foi submetido ao Comitê de Ética e Pesquisa (CEP) da Faculdade de Medicina de São José do Rio Preto, São Paulo, sob Protocolo CAAE 85890918.2.0000.5415 e aprovado em 12 de Abril de 2018, com o parecer no 2.595.328, atendendo as exigências legais para estudo envolvendo seres humanos.

\section{RESULTADOS E DISCUSSÃO}

Dos 51 pacientes participantes, a maioria era do sexo masculino, com idade igual ou superior a 60 anos, com média de idade de 54 anos e desvio-padrão de 16,3 anos, sem cônjuge/companheiro (a), cor branca autorreferida, sem conclusão do ensino médio, sem atividade remunerada e renda mensal de um a três salários mínimos (Tabela 1). 
Os resultados foram semelhantes aos encontrados, em outro estudo, em relação ao sexo, escolaridade e a não possuir cônjuge ${ }^{(10)}$.
Entretanto outros estudos encontraram predominância do sexo feminino e casados ${ }^{(11-12)}$.

Tabela 1 - Distribuição dos pacientes deficientes visuais diabéticos, segundo as características sociodemográficas. São José do Rio Preto, SP, Brasil, 2018. ( $n=51)$.

\begin{tabular}{|c|c|c|}
\hline Variáveis & $\mathbf{n}$ & $\%$ \\
\hline \multicolumn{3}{|l|}{ Sexo } \\
\hline Masculino & 30 & 58,8 \\
\hline Feminino & 21 & 41,2 \\
\hline \multicolumn{3}{|l|}{ Idade } \\
\hline 18 a 29 anos & 6 & 11,8 \\
\hline 30 a 39 anos & 3 & 5,9 \\
\hline 40 a 49 anos & 10 & 19,6 \\
\hline 50 a 59 anos & 10 & 19,6 \\
\hline 60 anos ou mais & 22 & 43,1 \\
\hline \multicolumn{3}{|l|}{ Estado Civil } \\
\hline Solteiro (a) & 12 & 23,5 \\
\hline Casado (a) & 21 & 41,2 \\
\hline Amasiado (a) & 1 & 2,0 \\
\hline Separado (a)/Divorciado(a) & 13 & 25,5 \\
\hline Viúvo (a) & 4 & 7,8 \\
\hline \multicolumn{3}{|l|}{ Cor (autodeclarada): } \\
\hline Branco (a) & 25 & 49,0 \\
\hline Pardo (a) & 21 & 41,2 \\
\hline Preto (a) & 5 & 9,8 \\
\hline \multicolumn{3}{|l|}{ Escolaridade: } \\
\hline Sem escolaridade/ Analfabeto & 1 & 2,0 \\
\hline Ensino Fundamental Incompleto & 24 & 47,1 \\
\hline Ensino Fundamental Completo & 4 & 7,8 \\
\hline Ensino Médio Incompleto & 2 & 3,9 \\
\hline Ensino Médio Completo & 13 & 25,5 \\
\hline Ensino Superior Incompleto & 1 & 2,0 \\
\hline Ensino Superior Completo & 6 & 11,8 \\
\hline \multicolumn{3}{|l|}{ Exerce atividade remunerada: } \\
\hline Não & 49 & 96,1 \\
\hline Sim & 2 & 3,9 \\
\hline \multicolumn{3}{|l|}{ Renda Mensal (em salários mínimo*): } \\
\hline$<1$ & 1 & 2,0 \\
\hline 1 a 3 & 31 & 60,8 \\
\hline 4 a 5 & 12 & 23,5 \\
\hline$>5$ & 7 & 13,7 \\
\hline
\end{tabular}

Fonte: Elaborada pelos autores.

*Salário Mínimo, Brasil, 2018: R\$ 954,00.

A cultura de invulnerabilidade e o estereótipo do homem criam resistência às práticas de autocuidado; os homens consideram que a procura pelo serviço de saúde demonstra fraqueza e insegurança, assim, procuram os serviços de saúde somente em situações emergenciais ${ }^{(13)}$. Considerando que uma das medidas primordiais de prevenção às complicações do DM é o autocuidado e o homem possui resistência à essa prática, pode-se justificar a maior prevalência do sexo masculino.

A etnia é considerada um fator de risco não modificável ao DM tipo $2^{(1)}$, entretanto, na última Pesquisa Nacional de Saúde (PNS), 
realizada, em 2013, pelo Instituto Brasileiro de Geografia e Estatística (IBGE), em parceria com o Ministério da Saúde, não foram observadas diferenças com significância estatística na prevalência do DM com a cor da pele ${ }^{(14)}$.

É possível observar uma convergência com os resultados alcançados por outros estudos, entre os quais $53,5 \%$ e $43,0 \%$ dos pacientes possuíam idade igual ou superior a 60 anos $^{(15-16)}$. A faixa etária de 60 a 69 anos, a baixa escolaridade e residir sozinho são fatores para um conhecimento deficiente sobre o DM. Também vale ressaltar que, antigamente, havia dificuldade de acesso à educação, o que pode justificar a baixa escolaridade dos idosos e o seu comparecimento frequente aos serviços públicos de saúde. Endo assim, é fundamental que os profissionais da saúde garantam a qualidade e a clareza nas orientações oferecidas sobre a doença e o tratamento a essa população ${ }^{(17)}$.

No Brasil, o Beneficio da Prestação Continuada (BPC) é garantido por lei e prevê um salário mínimo mensal ao idoso (acima de 65 anos) e ao portador de deficiência que comprovem não ter como se manter financeiramente, ou ser mantido por sua família. Esse benefício sofreu alterações desde a sua instituição, entretanto o limite de renda permanece o mesmo, ou seja, a renda mensal familiar per capita não pode ser superior a um quarto do salário mínimo ${ }^{(18)}$. Considerando que, na maioria das vezes, o mercado de trabalho oferta às pessoas com deficiência um rendimento igual ou inferior a que elas recebem com o benefício, muitos preferem não trabalhar e manter a renda assegurada a encarar discriminações e se expor ao ambiente de trabalho incerto e hostil ${ }^{(19)}$. Essas informações podem justificar e vão ao encontro dos resultados do presente estudo.

Os dados da Tabela 2 confirmam os dados da literatura, uma vez que a DM tipo 2, apesar de poder ser evitado, é o mais prevalente ${ }^{(1)}$.

Tabela 2 - Distribuição dos pacientes, segundo as características clínicas e estilo de vida. São José do Rio Preto, SP, Brasil, 2018. ( $\mathrm{n}=51)$.

\begin{tabular}{|c|c|c|}
\hline Variáveis & $\mathbf{n}$ & $\%$ \\
\hline \multicolumn{3}{|l|}{ Tipo de DM: } \\
\hline Tipo 1 & 23 & 45,1 \\
\hline Tipo 2 & 28 & 54,9 \\
\hline \multicolumn{3}{|l|}{ Tempo de descoberta do DM (em anos): } \\
\hline 1 a 3 & 7 & 13,7 \\
\hline 4 a 5 & 3 & 5,9 \\
\hline 6 a 10 & 12 & 23,5 \\
\hline$>10$ & 29 & 56,9 \\
\hline \multicolumn{3}{|l|}{ Antecedentes Familiares (pais e/ou irmãos): } \\
\hline Não & 20 & 39,2 \\
\hline Sim & 31 & 60,8 \\
\hline \multicolumn{3}{|l|}{ Tipos de tratamento: } \\
\hline Dieta & 1 & 2,0 \\
\hline Hipoglicemiantes orais & 12 & 23,5 \\
\hline Insulina & 4 & 7,8 \\
\hline Dieta e hipoglicemiantes orais & 16 & 31,4 \\
\hline Dieta e insulina & 14 & 27,5 \\
\hline Hipoglicemiantes orais e insulina & 1 & 2,0 \\
\hline Dieta, hipoglicemiantes orais e insulina & 3 & 5,9 \\
\hline \multicolumn{3}{|l|}{ Deixou de seguir o tratamento em algum momento: } \\
\hline Não & 31 & 60,8 \\
\hline $\operatorname{Sim}$ & 20 & 39,2 \\
\hline \multicolumn{3}{|l|}{ Dislipidemia: } \\
\hline Não & 27 & 52,9 \\
\hline Sim & 24 & 47,1 \\
\hline \multicolumn{3}{|l|}{ Praticava exercícios físicos regularmente: } \\
\hline Não & 40 & 78,4 \\
\hline Sim & 11 & 21,6 \\
\hline
\end{tabular}




\begin{tabular}{lcc}
\hline \hline \multicolumn{1}{c}{ Variáveis } & $\mathbf{n}$ & $\%$ \\
\hline Tabagista: & 48 & 94,1 \\
Não & 3 & 5,9 \\
Sim & & \\
Fazia uso de algum tipo de bebidas alcoólicas: & 42 & 82,4 \\
Não & 9 & 17,6 \\
Sim & & 25,5 \\
Hipertensão Arterial Sistêmica: & 13 \\
Não & 38 & 74,5 \\
Sim & 11 \\
Acompanhava na Atenção Primária à Saúde: & 40 \\
Não & & 21,6 \\
Sim & 78,4 \\
Tem/Teve acesso a atividades educativas de prevenção ao DM e suas complicações: & 39 \\
Não & 12 \\
Sim & & 76,5 \\
\hline \hline
\end{tabular}

Fonte: Elaborada pelos próprios autores.

Os resultados, também, corroboram os dados de outro estudo, cujo DM tipo 2 estava presente em $82,4 \%$ dos pacientes ${ }^{(15)}$. O tempo de diagnóstico do DM é uma variável de grande influência sobre a qualidade de vida, sendo que, em período prolongado, como dez anos, aumenta o risco de complicações ${ }^{(20)}$. Estudo realizado, no estado de Goiás, identificou, em sua amostra, que $51,4 \%$ dos pacientes foram diagnosticados com DM há mais de dez anos ${ }^{(15)}$. Em outro estudo, realizado em Minas Gerais, os dados mostraram que a presença de complicações relacionadas ao DM estava associada ao tempo de duração da doença, uma vez que os que possuíam o diagnóstico da doença, há mais de dez anos, tinham um percentual maior de complicações que aqueles com a doença há menos tempo ${ }^{(16)}$. Os resultados deste estudo indicam também esta realidade, visto que a maioria dos pacientes $(56,9 \%)$ descobriu a doença há mais de dez anos (Tabela 2).

Pesquisa realizada, na região Sul do Brasil, que analisou o perfil epidemiológico e avaliou a qualidade de vida de pessoas com DM, identificou dados de histórico familiar, mas, em percentuais menores. A maior prevalência dos fatores de risco foram o sedentarismo $(64,0 \%)$, dislipidemia (65,7\%) e sobrepeso/obesidade $(50,9 \%)$; menor prevalência foi o fator de risco tabagismo (20,3\%). Essa pesquisa, também, identificou que todos os pacientes realizavam algum tipo de tratamento ${ }^{(12)}$. Esses resultados corroboram os achados do presente estudo, entretanto nota-se que, apesar de todos os pacientes terem relatado fazer algum tipo de tratamento e a maioria nunca ter abandonado o tratamento, a dislipidemia, excesso de peso e o sedentarismo foram destaque na amostra estudada (Tabelas 2 e 3 ).

Aproximadamente um quarto dos pacientes tinha a HAS associada, dado semelhante a outros estudos ${ }^{(12,21)}$, nos quais a frequência de HAS também foi significante $(71,7 \%$ e $89,1 \%)$ entre os participantes.

Quanto à ingestão de bebidas alcoólicas, o resultado do presente estudo ficou acima $(17,6 \%$ dos participantes), quando comparado ao estudo realizado com dados provenientes do inquérito nacional de Pesquisa Dimensões Sociais das Desigualdades, os quais identificaram que $5,4 \%$ dos pacientes diabéticos ingeriam bebidas alcoólicas ${ }^{(2)}$.

As atividades de educação em saúde, visando ao controle do DM, devem ser consideradas parte do tratamento integral, estimando a dificuldade dos pacientes diabéticos em seguir o tratamento prescrito, tanto medicamentoso como não medicamentoso. Essas atividades aumentarão a compreensão sobre a doença, ajudarão no aumento da autonomia e na superação dos obstáculos diversos que dificultam o controle da doença, contribuindo, assim, para a prevenção das complicações associadas e à manutenção da qualidade de vida das pessoas portadoras de $\mathrm{DM}^{(22)}$. Estudo cujo objetivo era avaliar o conhecimento, a atitude e os fatores associados a pacientes com DM, na APS, identificou que $61,5 \%$ dos pacientes não participam de grupo de educação em $\mathrm{DM}^{(21)}$. Neste estudo, apesar da maioria relatar ter acompanhamento, na APS, apenas 23,5\% 
afirmaram que têm ou tiveram acesso às atividades educativas de prevenção ao DM e suas complicações (Tabela 2). Esse é um fator agravante, tendo em vista a necessidade e a importância de prevenir tal agravo e suas complicações.

Na Tabela 3, destacam-se os resultados do IMC dos pacientes, parte acima do peso e circunferência abdominal, acima do recomendado.
Verificou-se, também, que o tipo de DM não é um fator que influencia de forma significativa os resultados do IMC e a circunferência abdominal, visto que os valores " $p$ " resultaram superiores em nível de significância adotados (Tabela 3). Estudos têm enfatizado que os padrões alimentares associados ao sedentarismo podem ser os responsáveis pelo aumento da incidência e da prevalência da obesidade e do diabetes ${ }^{(22)}$.

Tabela 3 - Análise bivariada, relacionada ao estado nutricional e tipo de DM, nos pacientes deficientes visuais. São José do Rio Preto, SP, Brasil, 2018. (n=51).

\begin{tabular}{|c|c|c|c|c|c|c|c|}
\hline \multirow{2}{*}{ Variáveis } & \multicolumn{2}{|c|}{ DM Tipo 1} & \multicolumn{2}{|c|}{ DM Tipo 2} & \multicolumn{2}{|c|}{ Total } & \multirow{2}{*}{ p } \\
\hline & n & $\%$ & $\mathrm{n}$ & $\%$ & $\mathbf{n}$ & $\%$ & \\
\hline \multicolumn{8}{|l|}{ IMC } \\
\hline Baixo Peso & 1 & 4,35 & 0 & 0,00 & 1 & 1,96 & \multirow{6}{*}{$0,094^{*}$} \\
\hline Eutrófico & 3 & 13,04 & 9 & 32,14 & 12 & 23,53 & \\
\hline Sobrepeso & 10 & 43,48 & 12 & 42,86 & 22 & 43,14 & \\
\hline Obesidade I & 8 & 34,78 & 5 & 17,86 & 13 & 25,49 & \\
\hline Obesidade II & 0 & 0,00 & 2 & 7,14 & 2 & 3,92 & \\
\hline Obesidade III & 1 & 4,35 & 0 & 0,00 & 1 & 1,96 & \\
\hline \multicolumn{8}{|l|}{ Circunferência Abdominal } \\
\hline$<=90=$ Masculino/Normal & 3 & 13,04 & 6 & 21,43 & 9 & 17,65 & \multirow{4}{*}{$0,245^{*}$} \\
\hline$>=91=$ Masculino/Alterado & 9 & 39,13 & 12 & 42,86 & 21 & 41,18 & \\
\hline$<=80=$ Feminino $/$ Normal & 0 & 0,00 & 1 & 3,57 & 1 & 1,96 & \\
\hline$>=81$ = Feminino/Alterado & 11 & 47,83 & 9 & 32,14 & 20 & 39,22 & \\
\hline
\end{tabular}

Fonte: Elaborada pelos autores.

* Aplicado teste de Regressão de Poisson a p<0,05.

A maior parte dos pacientes diabéticos era cega e relatou que as doenças oculares (Retinopatia diabética (RD), glaucoma e catarata) foram a causa da DV, entretanto tais evidências não foram confirmadas de forma estatisticamente significante (Tabela 4).
Analisando a amostra por tipo de DM, destaca-se que a maioria dos pacientes diabéticos tipo 1 tornaram-se deficientes visuais, há menos de seis anos e os tipo 2 tornaram-se deficientes visuais há mais de seis anos, com associação estatisticamente significante entre o tipo de diabetes e o tempo da DV (Tabela 4).

Tabela 4 - Distribuição do tipo, tempo e causa da DV, segundo o tipo de DM referida pelos pacientes deficientes visuais. São José do Rio Preto, SP, Brasil, 2018. ( $n=51)$.

\begin{tabular}{|c|c|c|c|c|c|c|c|}
\hline \multirow{2}{*}{ Variáveis } & \multicolumn{2}{|c|}{ DM Tipo 1} & \multicolumn{2}{|c|}{ DM Tipo 2} & \multicolumn{2}{|c|}{ TOTAL } & \multirow{2}{*}{$\mathbf{p}$} \\
\hline & $\mathbf{n}$ & $\%$ & $\mathbf{n}$ & $\%$ & $\mathbf{n}$ & $\%$ & \\
\hline \multicolumn{8}{|l|}{ Tipo de DV: } \\
\hline Baixa Visão & 6 & 26,09 & 8 & 28,57 & 14 & 27,45 & \multirow[t]{2}{*}{$0,841^{*}$} \\
\hline Cego & 17 & 73,91 & 20 & 71,43 & 37 & 72,55 & \\
\hline \multicolumn{8}{|l|}{ Tempo da DV (em anos): } \\
\hline$<2$ & 9 & 39,13 & 5 & 17,86 & 14 & 27,45 & \multirow{2}{*}{$0,001^{*}$} \\
\hline 3 a 5 & 3 & 13,04 & 5 & 17,86 & 8 & 15,69 & \\
\hline
\end{tabular}




\begin{tabular}{|c|c|c|c|c|c|c|c|}
\hline \multirow{2}{*}{ Variáveis } & \multicolumn{2}{|c|}{ DM Tipo 1} & \multicolumn{2}{|c|}{ DM Tipo 2} & \multicolumn{2}{|c|}{ TOTAL } & \multirow{2}{*}{$\mathbf{p}$} \\
\hline & $\mathbf{n}$ & $\%$ & $\mathrm{n}$ & $\%$ & $\mathbf{n}$ & $\%$ & \\
\hline 6 a 8 & 4 & 17,39 & 1 & 3,57 & 5 & 9,80 & \\
\hline 9 a 11 & 2 & 8,70 & 3 & 10,71 & 5 & 9,80 & \\
\hline$>12$ & 5 & 21,74 & 14 & 50,00 & 19 & 37,25 & \\
\hline \multicolumn{8}{|l|}{ Causa/doença que levou a DV: } \\
\hline Doenças Oculares (RD, glaucoma e catarata) & 19 & 82,61 & 13 & 46,43 & 32 & 62,75 & \multirow{2}{*}{$0,389^{*}$} \\
\hline Outras & 4 & 17,39 & 15 & 53,57 & 19 & 37,25 & \\
\hline
\end{tabular}

Fonte: Elaborada pelos autores.

* Aplicado teste de Regressão de Poisson a p<0,05.

Estudo realizado, em ambiente hospitalar, no país de Gana, identificou que $18,4 \%$ dos pacientes diabéticos, que frequentavam a instituição, possuíam algum tipo de DV, sendo que $5,8 \%$ eram $\operatorname{cegos}^{(23)}$. Na Hungria, foi observado que $13,8 \%$ dos pacientes diabéticos com idade igual ou superior a 50 anos possuíam algum tipo de DV e $0,9 \%$ eram $\operatorname{cegos}^{(24)}$. Pesquisa realizada no México mostrou que $14,9 \%$ dos pacientes diabéticos, com mais de 50 anos, possuíam algum tipo de DV e $1,5 \%$ eram cegos $^{(25)}$. No Brasil, não foi localizado estudo recente envolvendo esta temática e, no presente estudo, todos os pacientes eram DV diabéticos, sendo que a maioria era cega. Tal fato se justifica pelo local da pesquisa ser um serviço especializado de reabilitação de pessoas deficientes visuais.

Observa-se, na Tabela 5, uma associação estatisticamente significante entre o tipo de DM e a presença da $\operatorname{RD}(p=0,008)$. A maioria $(78,26 \%)$ dos pacientes diabéticos tipo 1 possuía a doença. Entretanto, apesar de não apresentar uma associação estatisticamente significante $(p=$
0,561), destaca-se que o glaucoma não foi relatado por $52,9 \%$ dos pacientes e não predominou em nenhum tipo de DM. A catarata, embora, também, não possua uma associação estatisticamente significante $(p=0,760)$, prevaleceu em $58,8 \%$ da amostra estudada, com predomínio em ambos os tipos de DM.

Este estudo mostrou uma associação estatisticamente significante entre o tipo de DM e a presença da RD e a maior parte dos diabéticos tipo 1 possuía RD (Tabela 5). A RD é a principal causa de perda de visão, em adultos, em idade ativa, atingindo mais de um terço de todos os portadores de $\mathrm{DM}^{(1)}$. Segundo as Diretrizes da Sociedade Brasileira de Diabetes (SBD), no mundo, estima-se que 93 milhões de diabéticos possuem algum grau de RD e, no Brasil, aproximadamente, quatro milhões de pessoas são portadoras da doença ${ }^{(3)}$. No estudo realizado na Hungria, a RD foi responsável por $28 \%$ de cegueira e $50 \%$ de DV grave entre os participantes portadores de DM com idade igual ou superior 50 anos $^{(24)}$.

Tabela 5 - Distribuição das doenças oculares segundo o tipo de DM. São José do Rio Preto, SP, Brasil, 2018. $(n=51)$.

\begin{tabular}{|c|c|c|c|c|c|c|c|}
\hline \multirow{2}{*}{ Variáveis } & \multicolumn{2}{|c|}{ DM Tipo 1} & \multicolumn{2}{|c|}{ DM Tipo 2} & \multicolumn{2}{|c|}{ Total } & \multirow{2}{*}{ p } \\
\hline & $n$ & $\%$ & $\mathrm{n}$ & $\%$ & $\mathrm{n}$ & $\%$ & \\
\hline \multicolumn{8}{|l|}{ Glaucoma } \\
\hline Não & 12 & 52,17 & 15 & 53,57 & 27 & 52,94 & \multirow{2}{*}{$0,561^{*}$} \\
\hline Sim & 11 & 47,83 & 13 & 46,43 & 24 & 47,06 & \\
\hline \multicolumn{8}{|l|}{ Catarata } \\
\hline Não & 8 & 34,78 & 13 & 46,43 & 21 & 41,18 & \multirow{2}{*}{$0,760^{*}$} \\
\hline Sim & 15 & 65,22 & 15 & 53,57 & 30 & 58,82 & \\
\hline \multicolumn{8}{|l|}{ RD } \\
\hline Não & 5 & 21,74 & 22 & 78,57 & 27 & 52,94 & \multirow{2}{*}{$0,008^{*}$} \\
\hline Sim & 18 & 78,26 & 6 & 21,43 & 24 & 47,06 & \\
\hline
\end{tabular}

Fonte: Elaborada pelos autores.

*Aplicado teste de Regressão de Poisson a p<0,05. 
Além da RD, a catarata e o glaucoma, ainda, são patologias frequentes e precoces em indivíduos portadores de $\mathrm{DM}^{(3)}$. No Brasil, em 2015, estimou-se que havia 350.000 pessoas cegas por catarata, com aumento de $20 \%$ à prevalência de casos novos a cada ano. O glaucoma é considerado a segunda maior causa de cegueira no mundo, a incidência estimada é de 1 a $2 \%$ da população, em geral, aumentando esse percentual, após os 40 anos de idade ${ }^{(5)}$. Neste estudo, não houve uma associação estatisticamente significante entre a catarata, glaucoma e os tipos de DM. O glaucoma não foi relatado pela maioria dos pacientes e não houve predomínio em nenhum tipo de DM. A catarata prevaleceu na amostra estudada (Tabela 5).

Considerando que o local onde aconteceu a coleta de dados atende 104 municípios e não realiza o tratamento clínico do DM, não foi possível e viável o acesso aos dados de prontuários dos pacientes, para corroborar as informações referidas na entrevista, como a evolução da doença e suas complicações, sendo esta uma limitação deste estudo.

\section{CONSIDERAÇÕES FINAIS}

Os resultados demonstraram que os pacientes deficientes visuais, diabéticos são, em sua maioria, do sexo masculino, idade igual ou superior a 60 anos, sem companheiro (a), cor branca, não concluíram o ensino médio e, apesar de não exercerem atividade remunerada, possuem renda mensal de um a três salários mínimos.

Houve a predominância do DM tipo 2, com diagnóstico há mais de dez anos, histórico de antecedentes familiares. Embora todos tenham relatado fazer algum tipo de tratamento e a maioria nunca ter deixado de segui-lo, quase metade possuía dislipidemia; o excesso de peso e o sedentarismo foram altamente prevalentes. Poucos relataram que têm ou tiveram acesso a atividades educativas de prevenção ao DM e suas complicações.

A maior parte dos pacientes é cega, relatou que as doenças oculares (RD, glaucoma e catarata) foram a causa da DV. Os pacientes diabéticos tipo 1 tornaram-se deficientes visuais, há menos de seis anos e os tipo 2 tornaram-se deficientes visuais há mais de seis anos. 0 estudo revelou, também, uma associação estatisticamente significante entre o tipo de DM e a presença de RD.
Desta forma, os resultados sugerem novos estudos visando contemplar outras dimensões da assistência, principalmente na APS em que se podem obter comparações, por meio de fontes de dados primários e secundários, buscando identificar achados e associações que auxiliem em estratégias mais eficazes envolvendo o DM e a DV.

Portanto os resultados deste estudo, a alta prevalência e a escassez de artigos, no Brasil e no mundo, que abordam a DM e os deficientes visuais, destacam a necessidade de maior vigilância dos gestores e profissionais de saúde sobre a temática. A caracterização clínica e sociodemográfica dos pacientes deficientes visuais norteiam o gerenciamento do cuidado e os programas educativos a serem desenvolvidos pelos profissionais de saúde, uma vez que as estratégias de controle das doenças sofrem diferentes influências, dependendo das características da população acometida.

\section{REFERÊNCIAS}

1- International Diabetes Federation (IDF). IDF Diabetes Atlas. 8th edn. Brussels: International Diabetes Federation; 2017.

2- Flor LS, Campos MR. Prevalência de diabetes mellitus e fatores associados na população adulta brasileira: Evidências de um inquérito de base populacional. Rev Bras Epidemiol. 2017;20(1):1629. DOI: $10.1590 / 1980-5497201700010002$

3- Sociedade Brasileira de Diabetes (SBD). Diretrizes da Sociedade Brasileira de Diabetes: 2017-2018. São Paulo: Clannad; 2017.

4- Malta DC, Bernal RTI, Iser BPM, Szwarcwald $\mathrm{CL}$, Duncan BB, Schmidt MI. Fatores associados ao diabetes autorreferido segundo a Pesquisa Nacional de Saúde, 2013. Rev Saúde Pública 2017;51(supl 1):1-12. DOI: 10.1590/s1518$\underline{8787.2017051000011}$

5- Avila M, Alves MR, Nishi M. As condições de saúde ocular no Brasil. São Paulo: Conselho Brasileiro de Oftalmologia; 2015.

6- Mosquera CFF, Souza SC, Souza WC, Nascimento ACSG, Schlesener AH. Avaliação do equilíbrio estático em crianças com deficiência visual (DV). InCantare 2018;9(1):9-23. Disponível em:

http://periodicos.unespar.edu.br/index.php/inca ntare/article/view/2337/pdf 86

7- Bourne RRA, Flaxman SR, Braithwaite $T$, Cicinelli MV, Das A, Jonas JB, et al. Magnitude, temporal trends, and projections of the global prevalence of blindness and distance and near 
vision impairment: A systematic review and meta-analysis. Lancet Glob Health 2017;5(9):888-97. DOI: $\quad$ 10.1016/S2214109X(17)30293-0

8- Secretaria de Direitos Humanos da Presidência da República (BR). Cartilha do Censo 2010: Pessoas com deficiência. Brasília: SDH PR/SNPD; 2012.

9- Associação Brasileira para o Estudo da Obesidade e da Síndrome Metabólica (ABESO). Diretrizes brasileiras de obesidade 2016. São Paulo: ABESO; 2016.

10- Lima LR, Funghetto SS, Volpe CRG, Santos WS, Funez MI, Stival MM. Qualidade de vida e o tempo do diagnóstico do diabetes mellitus em idosos. Rev Bras Geriatr Gerontol. 2018; 21(2):180-90. DOI: $10.1590 / 1981-$ 22562018021.170187

11- Santos EMS, Souza VP, Correio IAG, Correio EBS. Autocuidado de usuários com diabetes mellitus: Perfil sociodemográfico, clínico e terapêutico. Rev Fundam Care Online 2018;10(3):720-8. DOI: $10.9789 / 2175-$ 5361.2018.v10i3.720-728

12- Moreschi C, Rempel C, Siqueira DF, Backes DS, Pissaia LF, Grave MTQ. Estratégias Saúde da Família: Perfil/qualidade de vida de pessoas com diabetes. Rev Bras Enferm. 2018;71(6):2899-906. DOI: 10.1590/0034-7167-2018-0037

13- Lemos AP, Ribeiro C, Fernandes J, Bernardes K, Fernandes R. Saúde do homem: Os motivos da procura dos homens pelos serviços de saúde. Rev Enferm UFPE 2017;11(supl 11):4546-53. DOI: 10.5205/reuol.11138-99362-1-

SM.1111sup201714

14- Instituto Brasileiro de Geografia e Estatística (IBGE). Pesquisa Nacional de Saúde 2013: Percepção do estado de saúde, estilos de vida e doenças crônicas. Brasil, grandes regiões e Unidades da Federação. Rio de Janeiro: Instituto Brasileiro de Geografia e Estatística; 2014.

15- Amaral RT, Barbosa AM, Teixeira CC, Brandão LGVA, Afonso TC, Bezerra ALQ, et al. Conhecimento dos diabéticos frente à doença e orientações no autocuidado. Rev Enferm UFPE 2019;13(2):346-52. DOI: 10.5205/1981-8963v13i02a239077p346-352-2019

16- Cortez DN, Reis IA, Souza DAS, Macedo MML, Torres HC. Complicações e o tempo de diagnóstico do diabetes mellitus na atenção primária. Acta Paul Enferm. 2015;28(3):250-5. DOI: 10.1590/1982-0194201500042

17- Borba AKOT, Arruda IKG, Marques APO, Leal MCC, Diniz ADS. Conhecimento sobre o diabetes e atitude para o autocuidado de idosos na atenção primária à saúde. Ciênc Saúde Coletiva 2019;24(1):125-36. DOI: 10.1590/141381232018241.35052016

18- Duarte CMR, Marcelino MA, Boccolini CS, Boccolini PDMM. Proteção social e política pública para populações vulneráveis: Uma avaliação do Benefício de Prestação Continuada da Assistência Social - BPC no Brasil. Ciênc Saúde Coletiva 2017;22(11):3515-26. DOI: 10.1590/1413-812320172211.22092017

19- Neves-Silva P, Prais FG, Silveira AM. Inclusão da pessoa com deficiência no mercado de trabalho em Belo Horizonte, Brasil: Cenário e perspectiva. Ciênc Saúde Coletiva 2015;20(8)2549-58. DOI: 10.1590/141381232015208.17802014

20- Matias COF, Matias COF, Alencar BR. Qualidade de vida em idosos portadores de Diabetes Mellitus Tipo 2 atendidos em Unidades Básicas de Saúde de Montes Claros/MG. Rev Bras Qual Vida 2016;8(2):119-29. DOI: 10.3895/rbqv.v8n2.3841

21- Assunção CS, Fonseca AP, Silveira MF, Caldeira AP, Pinho L. Conhecimento e atitude de pacientes com diabetes mellitus da Atenção Primária à Saúde. Esc Anna Nery 2017;21(4):1-7. DOI: 10.1590/2177-9465-ean-2017-0208

22- Borges DDB, Lacerda JTD. Ações voltadas ao controle do Diabetes Mellitus na Atenção Básica: Proposta de modelo avaliativo. Saúde Debate 2018;42(116):162-78. DOI: 10.1590/01031104201811613

23- Lartey SY, Aikins AK. Visual impairment amongst adult diabetics attending a tertiary outpatient clinic. Ghana Med J. 2018;52(2):84-7. DOI: 10.4314/gmj.v52i2.4

24- Tóth G, Szabó D, Sándor GL, Nagy ZZ, Karadeniz $\mathrm{S}$, Limburg $\mathrm{H}$, et al. Diabetes and blindness in people with diabetes in Hungary. Eur J Ophthalmol. 2019;29(2):141-7. DOI: 10.1177/1120672118811738

25- Ramos AL, Bastar PAG, Lansingh VC, Gomez JAR, Fragoso VV, Arellano FAS, et al. Rapid assessment of avoidable blindness: Prevalence of blindness, visual impairment and diabetes in Nuevo Leon, Mexico 2014. Ophthalmic Epidemiol. 2018;25(5/6):412-8. DOI: $\underline{10.1080 / 09286586.2018 .1501498}$

Nota: Este artigo foi extraído da dissertação - Diabetes mellitus como fator de risco para as doenças oculares em pacientes deficientes visuais, apresentada ao Programa de Pós-Graduação Stricto Sensu em Enfermagem, da Faculdade de Medicina de São José do Rio Preto, em 2019. 
Recebido em: 06/07/2019

Aprovado em: 06/11/2019

Endereço de correspondência:

Evandro Scarso de Brito

Rua dos Lírios, 731 - Jardim dos Seixas

CEP: 15061-090 - São José do Rio Preto/SP - Brasil

E-mail: evandroscarsobrito@gmail.com 